\title{
Hemispherectomy and Diaschisis: Rapid Improvement in Cerebral Functions After Right Hemispherectomy in a Six Year Old Child
}

\author{
Aaron Smith \\ The Unversty of Michigan \\ Marion L. Walker and Garth Myers \\ Primary Children's Medical Center, Salt Lake City, Utah
}

\begin{abstract}
Neuroradiological, neurological, and neuropsychological studies before and after hemispherectomy for seizures of a six year old boy with left hemiplegia at birth revealed striking postoperative improvement. Selzures ceased and have not recurred. Preoperative EEG studies from seven months of age revealed increasing pathological involvement of the left as well as right hemisphere. Postoperatively, normal tracings over the left hemisphere documented marked and continung improvement. In contrast to markedly subnormal preoperatwe UISC-R $I Q s$, retest at 19 months postoperatively showed WISC-R IQs within the normal range, with simular improvement in other tests of cognitwe, motor and sensory functions. The arrest and reversal of the preoperative pattern of increasing deterioration and disappearance of pathological involvement of left as well as right hemisphere functions following surgery is consistent with the disappearance of diaschisis as described by von Monakow in 1914, and has concervably stgnificant clinical as well as theoretical implications
\end{abstract}

Until the development of hemispherectomy for glioma in adults by Dandy in 1928 and for intractable seizures in children by Krynauw in 1950, the established concepts of differences in the functions of the two cerebral

I his research was partially supported by NINCDS Grants \#2 R01 NS10089 and \#5 R01 NS24344.

Requests for reprints should be sent to Aaron Smith, PhD. The University of Michigan, 210 Collingwood, \#220, Ann Arhor, MI 48103 
hemispheres and of the seemingly anatomically identical structures that comprise each hemisphere were based on studies of children and adults with focal lateralized lesions. More recently, studies of a small number of patients with epileptogenic lesions in either or both hemispheres treated with commissurotomy for relief of seizures have given rise to numerous articles and books introducing new claims and definitions of mutually exclusive functions of the left and right cerebral hemisphere (Gazzaniga, 1970; Gazzaniga \& Hillyard, 1971; Jaynes, 1976).

The continuing diverse findings in initial and later effects of focal lateralized lesions on language, verbal and nonverbal reasoning and memory, and of sensory and motor functions of component lateralized hemispheric structures, however, reflect inherent ambiguities in such studies (Smith, 1972, 1975). Based on extensive clinicoanatomic correlation studies, von Monakow $(1911,1914)$ called attention to the frequency and variety of "distance effects" manifested in diverse transient and persisting effects of lateralized lesions. He introduced the term diaschisis to describe the phenomena. Subsequently, numerous studies have reported the radiation of distance effects of focal lateralized lesions disrupting the functions of anatomically intact remote mechanisms not only in the damaged hemisphere, but in the opposite "healthy" hemisphere as well.

Since the reviews by Smith $(1972,1975)$, reports of experimental and clinical studies (Ginsberg, Reivich \& Giandomenico, 1976; Markowitsch \& Pritzel, 1978; Slater, Reivich, \& Goldberg, 1976; West, 1978; West, Deadwyler, Cotman, \& Lynch, 1976) reflect continuing controversies on the validity of von Monakow's concepts and on the neurogenesis of diaschisis. Apart from other confounding factors described by Smith $(1962,1972,1975)$ and Smith and Sugar (1975), the subsequent development or recovery of functions in children and adults with diverse lateralized lesions or in experimental animals may reflect the roles of compensating mechanisms either within the damaged hemisphere, or in the opposite intact hemisphere, or both.

In patients with intact residual structures, hemispherectomy permitted comparisons of initial and later effects of removing a left or right hemisphere damaged in early life with effects of removing a hemisphere that had developed normally until the growth of a tumor in adulthood. The marked differences in initial and later effects of hemispherectomy in children and adults in accumulating studies since 1965 have been described in previous reports (Burklund \& Smith, 1977; Smith, 1962, 1966, 1969, 1972, 1975, 1976, 1977). However, regardless of the differences in their extent, recovery or development of functions following hemispherectomy that have been chronically depressed or arrested in development reflects the removal of the attenuating effects of pathologic influences radiated from the diseased hemisphere and disrupting the functions of the opposite hemisphere.

Since 1968, one of us (AS) has been studying initial and long term effects 
of hemispherectomy for intractable seizures based on comparisons of performance on the Michigan Neuropsychological Battery (Smith, 1975, 1983). In addition to the case reported here, this battery had been administered to 63 such patients one or more times at varying postoperative intervals. Thanks to the strong interest and collaboration of neurosurgeon Dr. Marion Walker, and pediatric neurologist Dr. Garth Myers, systematic preoperative and postoperative clinical neurological, radiological, electroencephalographic, and neuropsychological studies of a six year old boy have provided the most comprehensive documentation in this series of striking changes in initial and later effects of removal of a right hemisphere that had incurred a pre-, peri-, or early post-natal epileptogenic lesion.

\section{SUBJECT}

Summarizing earlier clinical studies by Dr. Myers and Dr. Waiker, the patient was born on $1 / 12 / 71$, with a left spastic hemiplegia. He subsequently required tendon transfers in both arm and leg. He gained the ability to walk independently using a short leg brace. His mother reported that at $2^{1 / 2}$ years of age, his speech was tested and he was found to be approximately one year ahead of his age.

Seizures began in 1974 at approximately three years of age. Despite attempts to control the grand mal and petit mal seizures with large doses of multiple medications (including phenobarbitol, tegretol, valium, dilantin, and diamox), they increased in frequency and severity. Following onset and continuation of seizures, speech and learning steadily regressed and the patient was classified as retarded.

Repeated EEG studies beginning on $9 / 8 / 71$ at seven months of age initially showed slight abnormalities over the right hemisphere, followed by the emergence of spike and wave patterns, and gradually increasing abnormalities after the first seizure in 1974. As the frequency and severity of the seizures increased, EEG abnormalities increased, spreading from over the right to over the left hemisphere.

A CT scan in September 1975 demonstrated an enlarged right ventricle with a shift towards the right side, indicating decreased volume of the right hemisphere. A repeat CT scan in April 1976 confirmed the initial findings and also indicated that the right ventricle had further enlarged and that the atrophy of the right hemisphere had progressed further since the $1975 \mathrm{CT}$ scan. Consistent with the overall pattern, in his preoperative examination one month before hemispherectomy, Dr. Walker noted continuing deterioration of speech and increasing mental retardation following onset of seizures, a common preoperative pattern among the 63 similar cases tested with the same battery since 1968 . 


\section{PROCEDURES}

Preoperative tests showed normal scores in the Peabody Picture Vocabulary (53/IQ 95) and Raven Matrices (14, above the 50th percentile), but a subnormal score in Memory for Unrelated Sentences (6, below the norms of four year old boys); and consistent with Dr. Walker's appraisal, subnormal WISC-R IQs (V 66, P 49, FS 54). In addition to the obvious left hemiplegia, however, Purdue Pegboard tests of manual dexterity revealed markedly subnormal performances with the nonhemiplegic, presumably "good" right hand (4/8 pegs placed in 30/60" intervals versus age norms of $9 / 17)$; and Single and Double Simultaneous (face-hand) Stimulation (SDSS) studies of somatosensory functions also revealed right- as well as left-sided sensory deficits.

As shown in the Table 1, the Michigan Neuropsychological Battery was administered 14 days before surgery when the patient was 5 years and 8 months old. The same battery was readministered at 6,12, and 19 months postoperatively. Comparisons of performances revealed striking and continuing improvement in "higher" (verbal and nonverbal cognitive), as well as "lower" (sensory and motor) level cerebral functions.

\section{RESULTS}

In addition to cessation of seizures, follow-up studies showed rapid and continuing improvement in overall cerebral functions. Compared with preoperative performances, reexamination at 19 months postoperatively showed WISC-R IQs (V 96, P 87, FS 90), reflecting gains of 30 points in the FSIQ; associated with gains in Visual Organization (11 to 20); Raven Matrices (14 to 19); Symbol Digit written substitutions ( 0 to 18$)$ and oral substitutions (0 to 27); Peabody Picture Vocabulary (53 to 62); and Memory for Uni elated Sentences (6 to 13). Purdue Pegboand tests of manual dexterity also revealed striking improvement in right hand dexterity (from $4 / 8$ to $9 / 17$ pegs placed in $30 / 60^{\prime \prime}$ intervals); and SDSS tests of somatosensory functions revealed no signs of the right-sided deficits demonstrated in preoperative studies. Dr. Myers' EEG studies also showed disappearance of spike and wave patterns, and normalization of the tracings over the left hemisphere. Consistent with the postoperative clinical studies by Dr. Walker and Dr. Myers, and neuropsychological studies, the parents reported remarkable improvement in behavior and affect.

The rate of future mental development and ultimate intellectual competence that may be achieved as an adult by our patient cannot be reliably assessed. Selected studies reviewed in previous reports (Smith 1962, 1972, 1975) have suggested that the drastically reduced neuroanatomical economy following hemispherectomy for early epileptogenic lesions necessarily results in limitations of developmental language potentials after left hemi- 


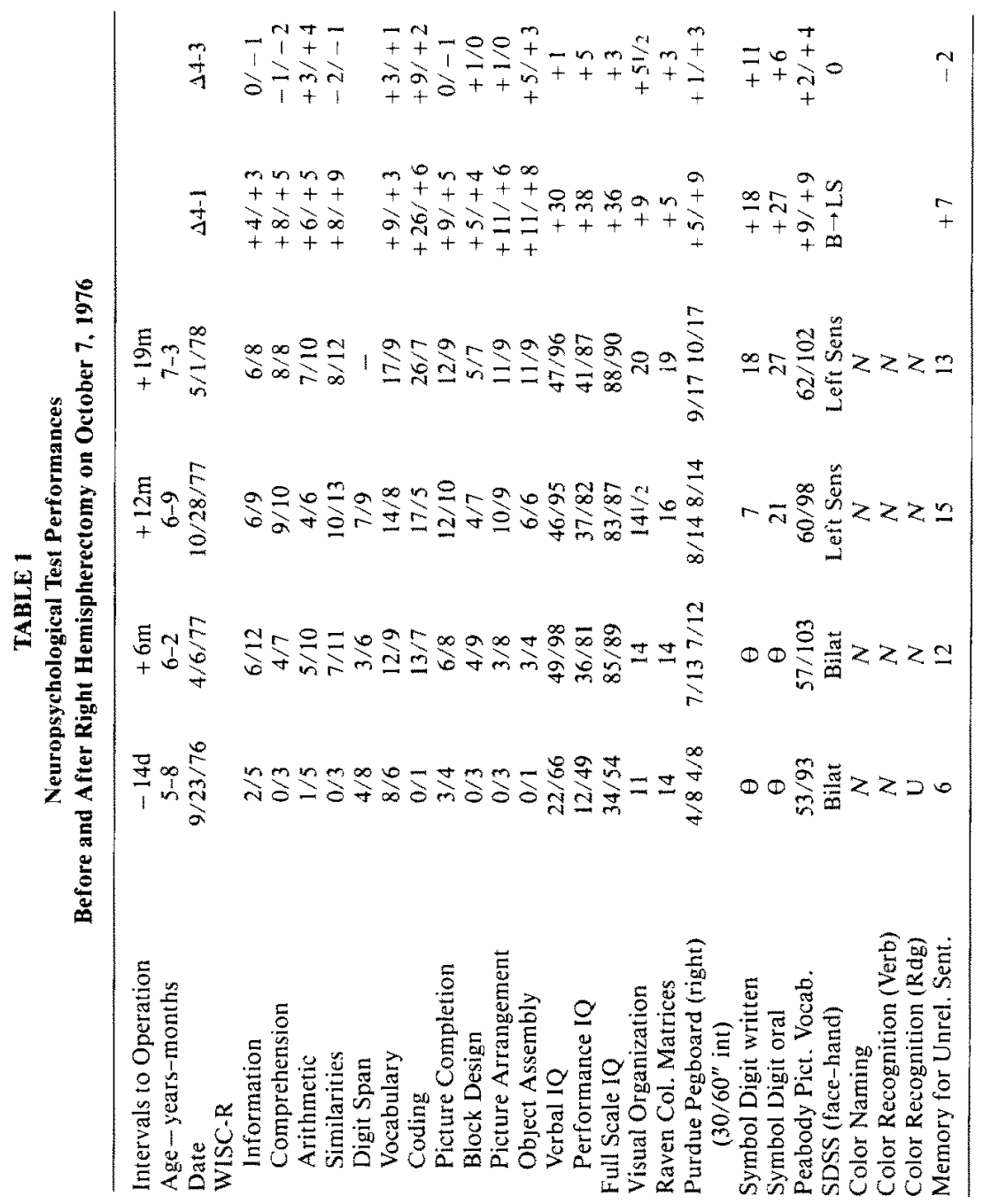


spherectomy and of nonlanguage functions after right hemispherectomy. Presumably, the left hemisphere was "meant to" specialize in language and verbal functions, and the right hemisphere was similarly consigned by nature to specialize in nonverbal functions. However, we may note that while the WISC-R Verbal IQ increased from 66 preoperatively to 96 only 19 months after right hemispherectomy, the corresponding increase in the Performance IQ from 49 to 87 was even larger. Moreover, the remarkable potentials for continuing development of superior adult language and intellectual capacities $15^{1 / 2}$ and 21 years after removal of the left hemisphere in a $51 / 2$ year old boy with a similar history of lateralized brain insult and intractable seizures have been documented by Smith and Sugar (1975).

The pattern of progressive deterioration of language, mental, sensory, and motor functions following onset of seizures in both cases was described by Krynauw (1950) as the major impetus for his development of the radical procedure of hemispherectomy. The similar patterns of arrest of the preoperative deterioration and of remarkable and continuing development of both verbal and nonverbal mental functions as well as sensory and motor functions 19 months after right hemispherectomy in our patient, and 21 years after left hemispherectomy have important theoretical as well as practical clinical implications.

Immediately, they emphasize the important differences between initial and later effects of the two categories of brain lesions described by Jackson (1873) as destroying and discharging lesions in diagnostic studies of children and adults with suspected disease or injury of the brain and in studies of populations with confirmed lateralized lesions. However, perhaps of greater importance, they demonstrate the significant enhancement of capacities in verbal and nonverbal cognitive as well as sensory and motor functions of the residual intact hemisphere and other remaining neural structures once they are freed from the disruptive pathologic influences radiated from a damaged hemisphere. (Since the same anticonvulsants were continued after surgery, the depressed functions before surgery cannot be readily attributed to the effects of medication.) They also demonstrate that "distance effects" or diaschisis in cases with lateralized cerebral lesions may persist for years and chronically depress the functions of the opposite anatomically healthy hemisphere and the brain as a whole. In view of the recently reported controversial findings in studies of lateralized lesions in clinical populations and of carefully designed ablations in animal experiments, it is also important to note that the findings in preoperative and continuing postoperative studies of effects of hemispherectomy for infantile epileptogenic lesions from Krynauw's report in 1950 to the present study have provided consistent and compelling evidence supporting von Monakow's descriptions of "distance effects" and diaschisis.

Obviously, the generalizability of our findings based on studies of a 
single case is limited. However, the rapid and continuing mental development after hemispherectomy at the age of six years for perinatal epileptogenic lateralized lesions is also consistent with previous reports that "the earlier the brain is damaged, or the more immature the encephalization, the more marked is the compensation of function that may be expected" (Ueki, 1966, p. 332).

Finally, in contrast to earlier findings, Hecaen and Albert cite selected studies in support of claims that "... hemispheric specialization is established at an extremely young age and may even be innate," and that ". . . unilateral lesions in the neonatal or perinatal period reveal hemisphere-specific deficits that are similar to those found in adults with unilateral brain damage" (Hecean and Albert, 1978, p. 417); or, in other words, that the plasticity of the immature brain has been "overrated." However, the striking improvement and continuing development of both nonverbal and verbal intellectual capacities after right hemispherectomy in our patient, and similar patterns documented in others with left as well as right hemispherectomy (Smith, 1972, 1976, 1977; Smith \& Sugar, 1975) are consistent with earlier findings and demonstrate the remarkable plasticity of the infant and young brain. In addition to the continuing diminution of the severity of language, verbal and nonverbal cognitive, motor and sensory deficits in serial postoperative studies, the findings suggest that many of the continuing diverse and seemingly contradictory findings in studies of effects of lateralized cerebral lesions in children and adults may be reconciled when diaschisis and time as well as age and other factors are taken into account.

\section{REFERENCES}

BurkIund, C. W., \& Smith, A. (1977). Language and the cerebral hemispheres: Observations of verbal and non-verbal responses during eighteen months following left (dominant) hemispherectomy. Neurology, 27, 627-733.

Dandy, W. E. (1928). Removal of right cerebral hemisphere for certain tumors with hemiplegia: Preliminary report. Journal of the American Medical Association, 90, 823-825.

Gazzaniga, M. (1970). The bisected brain. New York: Appleton-Century-Crofts.

Gazzaniga, M., \& Hillyard, S. (1971). Language and speech capacity of the right hemisphere. Neuropsychologıa, 9, 273-280.

Ginsberg, M. D., Reivich, M., \& Giandomenico, A. (1976). Alterations of regional brain metabolism during focal ischemia. Neurology, 26, 346.

Hecaen, H., \& Albert, M. L. (1978). Human neuropsychology. New York: John Wiley \& Sons. Jackson, J. H. (1873). On the anatomical and psychological localization of movements in the brain. Lancet. In J. Taylor (Ed.). (1958): Selected writings of John Hughlings Jackson. London: Staples Press.

Jaynes, S. (1976). The origin of consciousness in the breakdown of the bicameral mind. Boston: Houghton Miflin.

Krynauw, R. A. (1950). Infantile hemiplegia treated by removing one cerebral hemisphere. Journal of Neurology, Neurosurgery \& Psychiatry, 13, 243-267. 
Markowitsch, H. J., \& Pritzel, M. (1978). Von Monakow's diaschisis concept: Comments on West et al. (1976). Behavoral Biology, 22, 411-412.

Monakow, C. von. (1911). Localization of brain functions. Journal for Psychologie und Neurologle, 17, 185. In G. von Bonin (Transl. 1960). Some papers on the cerebral cortex. Springfield, IL: Charles C. Thomas.

Monakow, C. von (1914). Die Lokalization im Grosshirn und der Abbau der Funktion durch Kortakale Herde. Wiesbaden: J. F. Bergniann.

Slater, R., Reıvich, M., \& Goldberg, H. 1. (1976) Cerebral diaschisıs in stroke. Stroke, 7, 7-8.

Smith A. (1962). Ambiguities in concepts and studies of 'brain damage' and 'organıcity.' Journal of Nervous and Mental Disease, 135, 311-326.

Smith, A. (1966). Speech and other functions after left (dominant) hemispherectomy. Journal of Neurology, Neurosurgery \& Psychiatry, 29, 467-471.

Smith, A. (1969). Nondomınant hemispherectomy. Neurology, 19, 442-445.

Sinith, A. (1972). Domunant and nondominant hemispherectomy. In W. Lynn Smith (Ed.), Drugs, development, and cerebral function. Springfield, IL: Charles C. Thomas.

Smith, A (1975). Neuropsychological testıng in neurological disorders. In W J. Friedlander (Ed.), Advances in neurologv (Vol. 7). New York: Raven Press.

Smith, A. (1976). Differing effects of hemispherectomy in children and adults $\mathrm{p}$ 217. Amer1can Psychological Associatıon 84th Annual Convention Abstracts, Washington, DC.

Smith, A. (1977). Language and nonlanguage functions after right or left hemispherectomy for cerebral lesions in infancy. 5th Annual Meeting, International Neurospychological Sociery, Santa Fe, NM. INS Bulletin Abstracts, p. 5.

Smith, A. (1983). Clinical psychological practice and principles of neuropsychological assessment. In C. Eugene Walker (Ed.), Handbook of clinical psvchology. Homewood, IL· Dow Jones-Irwin.

Smith, A., \& Sugar, O. (1975). Development of above normal language and intelligence 21 years after left hemispherectomy. Neurology, 25, 813-818.

West, J. R. (1978). The concept of diaschisı: A reply to Markowitsch and Pritzel. Behavoral Bıology, 22, 413-416.

West, J. R., Deadwyler, S. A., Cotman, C. W., \& Lynch, G S (1976). An experimental test of diaschisıs. Behavoral Bıology, 18, 419-425

Uek1, K. (1966). Hemispherectomy in the human with special reference to the preservation of function In T. Tokızane \& J. P. Schade (Eds.), Progress in brain research (Vol. 21B). New York: Elsevier. 